\title{
Ratio of dielectric strength of structural and secondary relaxation close to the glass transition for PPGE and DGEBA
}

\author{
Soheil Sharifi ${ }^{1^{*}}$, Nima Bahreini ${ }^{2}$ \\ ${ }^{1}$ Department of Physics, University of Sistan and Baluchestan, Zahedan, Iran; *Corresponding Author: soheil.sharifi@gmail.com \\ ${ }^{2}$ Department of Physics, University of Emam Khomeini, Nowshahr, Iran
}

Received 9 March 2012; revised 13 April 2012; accepted 28 April 2012

\begin{abstract}
Here, dielectric spectroscopy is used in the frequency range from $10^{-2} \mathrm{~Hz}$ up to $10^{7} \mathrm{~Hz}$ and we found dynamics of the primary $\alpha$ - and intermolecular Johari-Goldstein $\beta$-processes are strongly correlated in diglycidyl-ether of bis-phenol-A and poly phenyl glycidyl-ether (PPGE) over a wide temperature from 193 to $345 \mathrm{~K}$ and pressure $P$ range from 0.1 to $600 \mathrm{MPa}$. In contrast with the widespread opinion of statistical independence of these processes the $\alpha-\beta$ mutual dependence is quantitatively confirmed in [1] analysing the temperature and pressure behavior of the $\alpha$ - and (JG) $\beta$-processes the investigation of the ratio of dielectric strength of two processes close to $\mathrm{Tg}$ evidence that the importance of secondary dynamics in relaxing external electric stresses increase in glass formers at high pressures with respect to that of the structural relaxation. We suggest that the thermal agitation, acting above $T_{g}$ is at the basis of the observed result.
\end{abstract}

Keywords: Dielectric Strength; Glass Transition; PPGE; DGEBA; Pressure and Temperature

\section{INTRODUCTION}

The glassy state is a non equilibrium state of materials [2]. As a consequence, the observed thermodynamic and relaxation properties slowly evolve with time because of the evolution of the molecular configuration towards a state with lower energy [3]. The relaxation strength, $\Delta \varepsilon$, of the secondary process was observed to decrease [4,5] or to vary non monotonously [6,7] with time at fixed thermodynamic conditions in the glassy state. Another consequence of the non equilibrium condition of glasses is that thermodynamic and relaxation properties show different values when measured data's in the glassy state at fixed thermodynamic condition subsequently to different vitrification histories [8-10]. For example, this was observed for the relaxation frequency of several secondary processes $[4,11]$ consequently to cooling with different rates, or after vitrification combining different sequences of cooling and compression steps [12-14]. The reason why secondary processes are influenced by the thermodynamic history of the glass is not clear at all, and the microscopic mechanisms at the basis of such dependence are unknown. The situation is even more complicated since the molecular mechanisms at the basis of secondary process are not clear at all. Some secondary relaxations are interpreted in terms of small rotations of molecular subgroups decoupled from the whole molecule [15]. Another interpretation is that secondary processes originate from local, non-cooperative, reorientation of the whole molecule [15-17]. A route to distinguish JG and non-JG processes on the basis of their dynamic properties is reported on previous publications [17,18]. A greater sensitivity to the thermodynamic history is expected for secondary processes of JG type due to their intermolecular origin Glass forming materials are characterized by a complex relaxation pattern, which evolves over several decades. Dielectric spectroscopy is particularly efficient for such studies since it is able to monitor the dielectric dynamics of a system over a range up to 16 decades. It is challenging to distinguish between intermolecular and intramolecular $\beta$-processes also in systems with a complex molecular structure, even showing more than on $\beta$-process. Intermolecular $\beta$-processes observed in rigid molecule is called Johari-Goldstein $\beta$ processes (JG-processes). Among secondary relaxations, those originated by intra-molecular transitions naturally bear no relation to the structural relaxation. However, some relation may be expected for intermolecular secondary relaxations [19-21], originated by local motions of the entire molecule. These processes, possibly relevant for the glass transition, are now referred to as the Johari-Goldstein (JG) or $\beta$-relaxation to honor their discov- 
ery of secondary relaxation even in totally rigid molecules without internal degree of freedom. A rational of relaxation dynamics in such terms is for example proposed by the Coupling Model [22].

The relationship between structural and secondary dynamics is one of the most recently considered and possibly important issues of the dynamics in glass formers. One relation of interest is the ratio between the dielectric strength of the secondary processes, where $T_{g}$ is the glass transition temperature. The dielectric strength is the maximum electric field strength that depends on the configuration of the material or the electrodes with which the field is applied. The ratio of dielectric strength is the ratio of density of dipole moment of alpha to the secondary relaxation that it seems depends to the density and structure of materials. In this work we study by dielectric spectroscopy the relation dielectric strength of structural and the secondary processes in the poly phenyl glycidyl-ether (PPGE) and diglycidyl-ether of bisphenolA (DGEBA) in an interval of pressure ranging from 0.1 to $600 \mathrm{MPa}$ and temperature from 345 to $233 \mathrm{~K}$ at glass transition temperature. In the both systems, $\Delta \varepsilon_{\alpha} / \Delta \varepsilon_{\beta}$ decreases as pressure increases. The value of $\Delta \varepsilon_{\alpha} / \Delta \varepsilon_{\beta}$ at glass transition temperature for DGEBA is higher than PPGE.

\section{EXPERIMENT}

\subsection{Methods}

Dielectric measurements were carried out by a dielectric spectrometer (Alpha-Novocontrol) in the frequency interval of $10^{-2}-10^{7} \mathrm{~Hz}$. For measurements at ambient pressure the sample was placed in a parallel plate cell (diameter $30 \mathrm{~mm}$, gap $0.1 \mathrm{~mm}$ ) and the temperature is controlled at a precision of $0.1 \mathrm{~K}$ by using a dry-nitrogen stream based system. For measurements at high pressure the sample was placed in a parallel plate cell (diameter $20 \mathrm{~mm}$, gap $0.05 \mathrm{~mm}$ ) that, properly insulated from the external environment, was located inside a pressure chamber. Pressure variations $(0.1-600 \mathrm{MPa})$ were generated by a manual pump and transmitted to the sample through silicon oil. Additionally, a liquid circulator that is connected to a jacket and wrapped around the pressure room, allowed the control of temperature (353 - $233 \mathrm{~K})$ within $0.1 \mathrm{~K}$.

\subsection{Materials}

Poly [(phenyl glycidyl ether)-co-formaldehyde] (PPGE) in the chemical formula Figure 1(a), with average molecular weight of $345 \mathrm{~g} / \mathrm{mol}$, and $T_{g}$ around $258 \pm 1 \mathrm{~K}$, [23], were supplied by Aldrich Chemicals. Poly (Bisphenol A-co-epichlorohydrin), glycidyl-ether capped (DGEBA), with average molecular weight of $\sim 380 \mathrm{~g} / \mathrm{mol}$, corresponding to $n=0.14$ in the chemical formula Figure

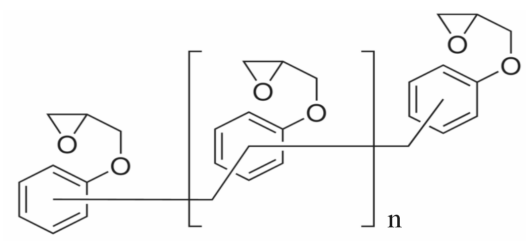

(a)

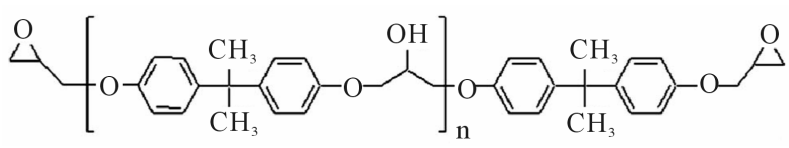

(b)

Figure 1. (a) Chemical structure of poly [(phenyl glycidyl ether)co-formaldehyde]; (b) Chemical structure of poly (BisphenolAcoepichlo rohydrin), glycidyl end-capped.

1(b), and glass transition temperature $255 \pm 1 \mathrm{~K}$ was purchased from Shell Co. under the trade name of Epon 828.

\section{RESULTS}

Dielectric loss spectra $\varepsilon(\omega)=\varepsilon^{\prime}-i \varepsilon^{\prime \prime}$, of PPGE, DGEBA were measured from above to below the glass transition. In Figure 2, the different thermodynamic paths are reported along which reinvestigated the relation between the structural and the secondary relaxation close to $T_{g}(P)$. Dashed lines show isothermal paths and solid lines show isobaric paths followed during the vitrification. For all the systems here investigated the secondary process was analysed only in the glassy state close and below $T_{g}$.

We acquired dielectric spectra along isothermal paths by varying pressure from $0.1 \mathrm{MPa}$ up to the maximum value of $600 \mathrm{MPa}$, with step of $10 \mathrm{MPa}$ in the supercooled liquid and step of $50 \mathrm{MPa}$ in the glass Figures 3 and 4. We also measured dielectric spectra by varying temperature at high pressure from 344.5 to $233 \mathrm{~K}$ with step of $3 \mathrm{~K}$ in supercooled liquid and $5 \mathrm{~K}$ in the glass (Figures 3 and 4 report some representative spectra). The increase of pressure slows down the structural relaxation similarly to the decrease of temperature. The structural relaxation peak shifts to lower frequencies until the structure of the liquid is eventually arrested in a glassy state. At ambient pressure in PPGE, DGEBA only the $\alpha$-structural process is visible above $T_{g}$, when spectra are collected in the frequency interval $10^{-2}-10^{6} \mathrm{~Hz}$. In the case of PPGE and DGEBA, for temperatures below $T_{g}$ two further relaxation processes, $\beta$-and, $\gamma$-are visible in the frequency interval Figures 3 and 4 . All the observed relaxation processes move towards lower frequencies on decreasing temperature or increasing pressure, the $\alpha$-process being the most sensitive and the $\gamma$-the less. The $\gamma$-process occurs only at very high frequency and in our frequency window can be measured only at very low temperature at ambient pressure, but 


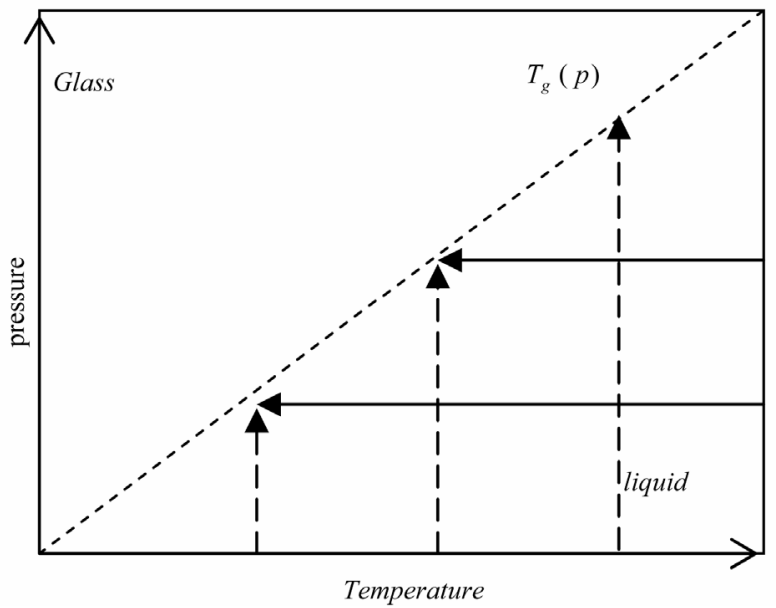

Figure 2. Schematic representation of the thermodynamic paths used during the measurements reported in this chapter. Squares (green points) represent the glass transition temperature at different pressure. Dashed and continuous lines represent isothermal and isobaric paths to the glass transition.

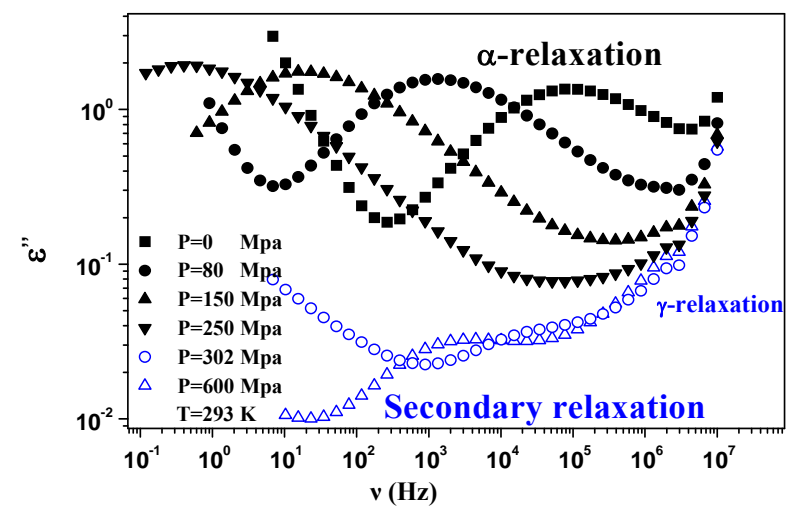

(a)

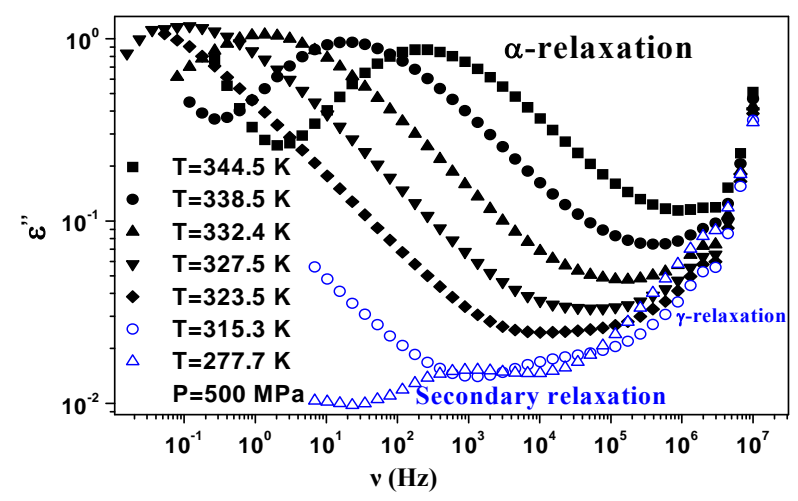

(b)

Figure 3. (a) Isothermal $(293 \mathrm{~K})$ dielectric loss spectra of PPGE, measured at different pressures (reported in the figure); (b) Isobaric (500 MPa) dielectric loss spectra of PPGE, measured at different temperatures reported in the figure). The black spectra are measured in the supercooled liquid and are dominated by the peak and the blue open symbols spectra are measured in the glassy state.

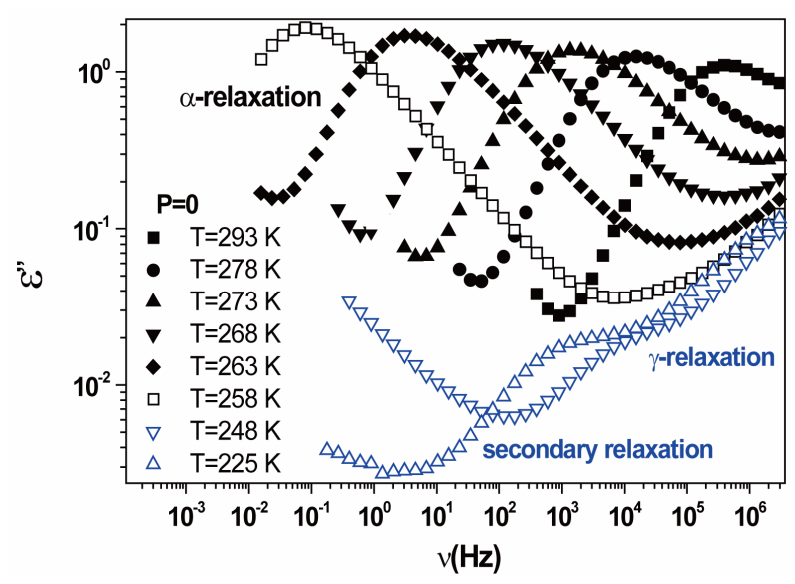

(a)

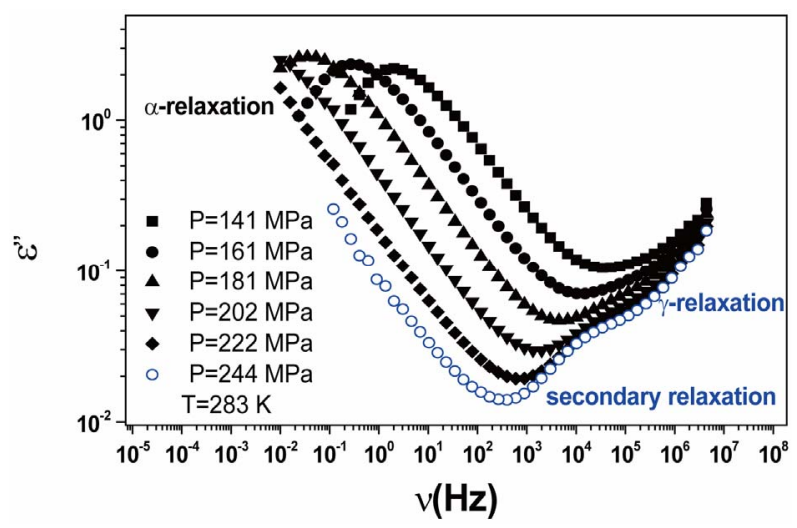

(b)

Figure 4. (a) Isobaric (0.1 MPa) dielectric loss spectra of DGEBA measured at different temperatures (values reported in the figure); (b) Isothermal (283 K) dielectric loss spectra of DGEBA measured at different pressure (values reported in the figure). The black spectra are measured in the supercooled liquid and are dominated by the $\alpha$-peak and the blue open symbols spectra are measured in the glassy state.

never at high pressure due to limitation of the temperature interval Figures 3 and 4.

Dielectric spectra were fitted with a superposition of different $\mathrm{HN}$ and $\mathrm{CC}$ functions, one for each relaxation process. Since for any value of temperature not more than two relaxation processes appear, in the fitting procedure we never used more than two relaxation functions. Moreover, the b-process was usually described by the $\mathrm{CC}$ function, and the contribution of the g-process at high pressure (for PPGE and DGEBA), was accounted by an $\mathrm{HN}$ function with only Deg and $T_{g}$ as free parameters, and the $a_{g}$ and $b_{g}$ parameters fixed to the values found at ambient pressure and very low temperature. The fitting program, based on a least square minimizing procedure, contemporary fits the real and the imaginary part of the dielectric spectrum and furnishes the values of the parameters and the corresponding errors. Tables $\mathbf{1}$ and $\mathbf{2}$ report 
Table 1. Parameters of $\alpha$-(structural), $\beta$-(secondary) relaxation at different pressures and temperatures for PPGE.

\begin{tabular}{cccccccc}
\hline & \multicolumn{3}{c}{$\alpha$-relaxation } & \multicolumn{2}{c}{$\beta$-relaxation } & \multicolumn{2}{c}{$\gamma$-relaxation } \\
\hline$P(\mathrm{Mpa})$ & $T(\mathrm{~K})$ & $\alpha$ & $\beta$ & $\alpha$ & $\beta$ & $\alpha$ & $\beta$ \\
\hline 0 & 293 & 0.35 & 0.49 & 0 & 0 & 0 & 0 \\
76 & 293 & 0.36 & 0.60 & 0 & 0 & 0 & 0 \\
150 & 293 & 0.39 & 0.67 & 0 & 0 & 0 & 0 \\
302 & 293 & 0.54 & 1.0 & 0.59 & 1.0 & 0 & 0 \\
600 & 293 & 0.38 & 0.96 & 0.67 & 1.0 & 0 & 0 \\
500 & 338 & 0.47 & 1.0 & 0 & 0 & 0 & 0 \\
500 & 335 & 0.49 & 1.0 & 0 & 0 & 0 & 0 \\
500 & 332 & 0.49 & 1.0 & 0 & 0 & 0 & 0 \\
500 & 315 & 0 & 0 & 0.62 & 1.0 & 0.30 & 0.32 \\
500 & 277 & 0 & 0 & 0.54 & 1.0 & 0.46 & 0.45 \\
\hline
\end{tabular}

Table 2. Parameters of $\alpha$-(structural), $\beta$-(secondary) relaxation at different pressure and temperature for DGEBA.

\begin{tabular}{cccccccc}
\hline & \multicolumn{3}{c}{$\alpha$-relaxation } & \multicolumn{2}{c}{$\beta$-relaxation } & \multicolumn{2}{c}{$\gamma$-relaxation } \\
\hline$P(\mathrm{Mpa})$ & $T(\mathrm{~K})$ & $\alpha$ & $\beta$ & $\alpha$ & $\beta$ & $\alpha$ & $\beta$ \\
\hline 240 & 293 & 0.24 & 0.6 & 0.6 & 1 & 0 & 0 \\
270 & 293 & 0.3 & 0.7 & 0.59 & 1 & 0 & 0 \\
300 & 293 & 0.47 & 0.81 & 0.48 & 1 & 0 & 0 \\
340 & 293 & 0.53 & 0.81 & 0.43 & 1 & 0 & 0 \\
141 & 283 & 0.28 & 0.64 & 0.51 & 1 & 0 & 0 \\
244 & 283 & 0 & 0 & 0.47 & 1 & 0.33 & 0.41 \\
303 & 283 & 0 & 0 & 0.44 & 1 & 0.37 & 0.43 \\
450 & 283 & 0 & 0 & 0.42 & 1 & 0.41 & 0.45 \\
0 & 273 & 0.15 & 0.43 & 0 & 0 & 0 & 0 \\
0 & 198 & 0.4 & 0.99 & 0.64 & 1 & 0 & 0 \\
\hline
\end{tabular}

some representative values of the parameters $a$ and $b$ for the considered systems at different pressure and temperature. Finally, since in all the cases, near $T_{g}$, the structural and the secondary relaxations are well separated in the frequency scale we reproduced the structural relaxation peak by the Fourier transform of the KWW function. This further analysis allowed a direct estimation of the stretching parameter characterizing the broadness of the structural peak Dielectric strength, $\Delta \varepsilon$, data of the structural and secondary relaxations are reported as a function of temperature and pressure in Figures 5 and 6. Above $T_{g}, \Delta \varepsilon_{\alpha}$ increase with increase pressure or decrease temperature. Below $T_{g}, \Delta \varepsilon_{\beta}$ remains

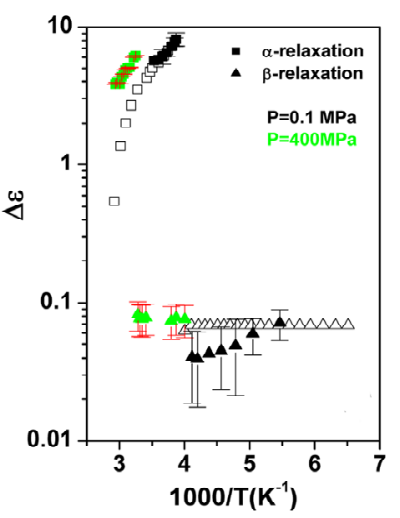

(a)

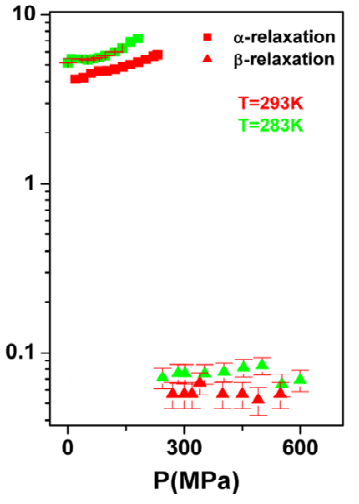

(b)
Figure 5. (a) Temperature and (b) pressure dependence of the dielectric strengths of DGEBA: $\Delta \varepsilon_{\alpha}$ (squares) and $\Delta \varepsilon_{\beta}$ (upward triangles). Open symbols represent data from Ref. [31].

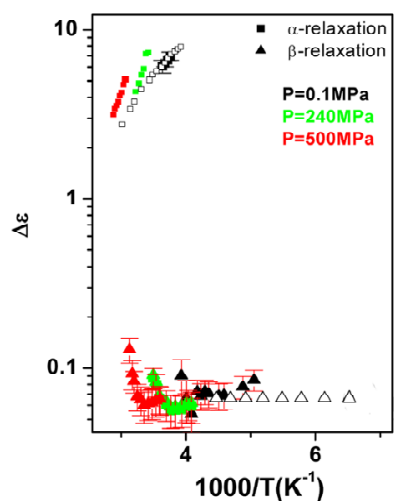

(a)

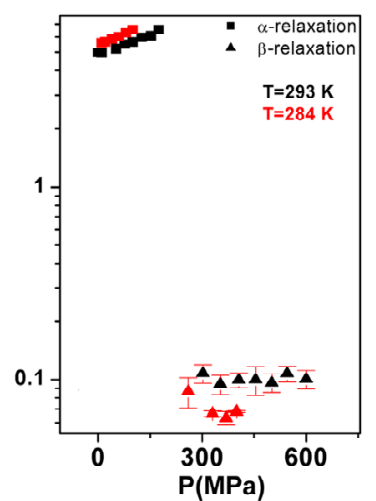

(b)
Figure 6. (a) Temperature and (b) pressure dependence of the dielectric strengths of PPGE: $\Delta \varepsilon_{\alpha}$ (squares) and $\Delta \varepsilon_{\beta}$ (upward triangle). Open symbols represent data from Ref. [31].

nearly constant in the investigated interval well inside the glassy state, but a definitive conclusion about its temperature dependence cannot be achieved due to the difficulty of the fit and the resulting uncertainty of the data. For PPGE, $\Delta \varepsilon_{\beta}$ close to glass transition temperature rapidly increases with temperature as already observed in many secondary relaxation processes. This behaviour is a clear indication of the influence of the glass transition phenomenon on the b-process of PPGE [24,25]. Unfortunately, due to the difficulties of the fit we did not observe the same scenario for the other two systems. For comparison we added in Figures 4 and 5 the dielectric strength data at ambient pressure for PPGE and DGEBA from refs [25], which are reported as open symbols. We can observe a good agreement among our data and those from literature. With the study of $\Delta \varepsilon_{\beta}$ of DGEBA we can observe some discrepancies that can be due to the difficulties of the fit The structural relaxation times for PPGE was already investigated with $M$. Paluch and 
co-workers, near $T_{g}$ over a wide range of thermodynamic variables (for example pressure, temperature and volume), [26]. This study showed that temperature is not the dominant control variable for structural relaxation.

Broadband dielectric spectroscopy, heat capacity spectroscopy, and viscosimetry have been used by S. Corezzi and co-workers to study PPGE at ambient pressure. In spite of their rather simple molecular structure, the dynamics of these systems is characterized by two wellseparated crossover regions where the relaxation times of structural relaxation and the two secondary relaxations $b$ and $\gamma$ approach each other [27].

The structural relaxation in diglycidylether of bisphenol A (DGEBA) has been examined studied with three spectroscopic methods: dielectric spectroscopy (DS), dynamic light scattering-photon correlation spectroscopy (LS), and mechanical spectroscopy (MS) [28-30]. The DS and LS measurements were carried out as a function of both temperature and pressure. Moreover, pressurevolume-temperature measurements were obtained for the DGEBA. These data allow an assessment of the relative contributions of thermal energy and free volume to structural relaxation in DGEBA. The results clearly show a substantial role for both thermal and free volume in the dramatic slowing down of the dynamics. The combined temperature- and pressure-dependences of the dielectric and light scattering relaxation times were analyzed using the Avramov equation, showing that the fragility (normalized temperature dependence) is pressure independent over the studied range of pressures. Conformance to the time-temperature-pressure superposition principle was also observed for all measurement techniques [31].

\section{DISCUSSION}

The dielectric strength data as a function of temperature and pressure are shown in Figures 5 (for DGEBA) and $\mathbf{6}$ (for PPGE). According to statistical theory of dielectric relaxation, the total dielectric strength, $\Delta \varepsilon_{\text {tot }}$, of an ensemble with dipole moments density, $N$, is proportional to the square value of the dipole moment, $\mu$, and can be approximated by $\Delta \varepsilon=g N \mu^{2} / 3 K_{B} T$. In this expression $K_{B}$ is the boltzman constant, and $g$ is the Kirkwood correlation factor, which takes into account the local, static correlation among the dipoles. It is usually reported that the temperature dependence of $\Delta \varepsilon_{\alpha}$ is similar as that of $\Delta \varepsilon_{\text {tot }}$ at least when the contribution from other relaxation processes is negligible. Our investigation evidences, that $\Delta \varepsilon_{\alpha}$, increases when the temperature decrease, approximately with $1 / T$ behavior, in agreement with the results reported in literature. Moreover, $\Delta \varepsilon_{\alpha}$ increases also linearly with pressure along isothermal paths in the supercooled liquid. This behavior is in part due to the increase with pressure of the dipole moments density, $N$, and to the decrease of the thickness of sample due to the compressibility of the material. The last effect causes a variation of the empty capacity of the cell that, when not correctly considered, led to an apparent increase of $\Delta \varepsilon_{\alpha}$. In the glassy state the effect of $P$ and $T$ on the dielectric strength of the secondary relaxation is not clear. Usually we observe that the two variables do not affect too much $\Delta \varepsilon_{\beta}$. However, this result can be again an artifact of the thickness variation of the sample during compression. The values of $\Delta \varepsilon_{\alpha}$ and $\Delta \varepsilon_{\beta}$ estimated at the glass transition are reported in Table 3. Since it is not possible to estimate the values of $\Delta \varepsilon_{\alpha}$ at $T_{g}$ they were calculated by extrapolation with the equation $\Delta \varepsilon_{\alpha}=A+B x$. For isobaric measurements $x$ is equal to $1000 / T$ (inverse of temperature) and for isothermal measurements $x$ is equal to $P$ (pressure). The values of the parameters $A$ and $B$ as calculated from the fitting of the available data are reported in Table 3.

Analogously, $\Delta \varepsilon_{\beta}$ was estimated with a linear extrapolation from the data close to $T_{g}$. In order to investigate any possible relation between $\alpha$ - and $\beta$-processes we studied the value of $\Delta \varepsilon_{\alpha} / \Delta \varepsilon_{\beta}$ at the glass transition for different thermodynamic conditions. This ratio, represent the amount of energy relaxed through the $a$-relaxation with respect to that relaxed by the $\beta$-one. In PPGE, $\Delta \varepsilon_{\alpha} / \Delta \varepsilon_{\beta}$, decreases when temperature and pressure are increased, though remaining at the glass transition Figure 7. The same behaviors as observed for DGEBA. In the case of DGEBA and PPGE, the $\Delta \varepsilon_{\alpha}$ and $\Delta \varepsilon_{\beta}$ are normalized with article that we mention before. This result evidences that the importance of secondary dynamics in relaxing external electric stresses increase in glass formers at high pressures with respect to that relaxed by the structural relaxation. A possible explanation of this phenomenon can be as follow. At high pressure

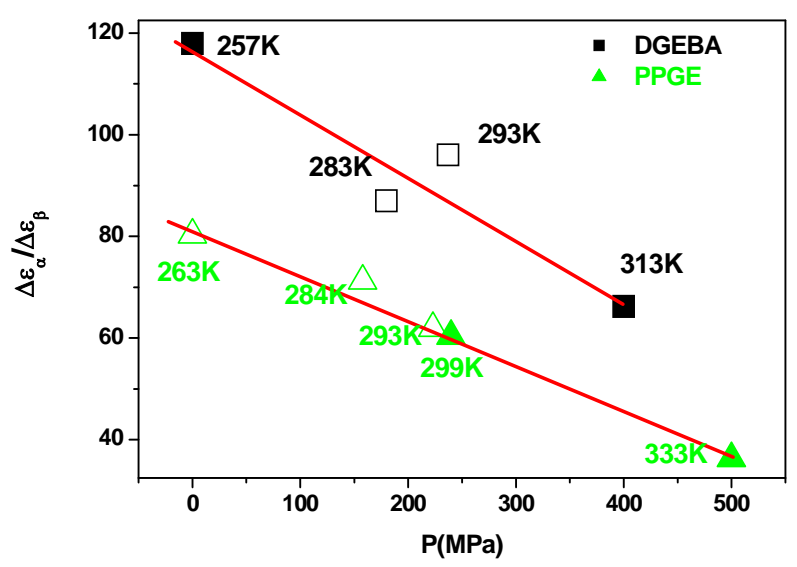

Figure 7. $\Delta \varepsilon_{\alpha} / \Delta \varepsilon_{\beta}$ as a function of pressure for DGEBA (square) and PPGE (up triangle), at glass transition temperature. Closed symbols refer to isobaric measurements, open symbols to isothermal ones. 
Table 3. Dielectric strength of $\alpha$ - and $\beta$-relaxation $\left(\Delta \varepsilon_{\alpha} / \Delta \varepsilon_{\beta}\right)$ at the glass transition temperature in PPGE, DGEBA. $A, B$ are the parameter used to reproduce the linear dependence of $\Delta \varepsilon_{\alpha}$ on $P$ or $1000 / T: \Delta \varepsilon_{\alpha}=A+B x, x=1000 / T$ or $x=P$.

\begin{tabular}{|c|c|c|c|c|c|c|c|}
\hline & $P(\mathbf{M P a})$ & $T(\mathbf{K})$ & $\log \left(v_{0 \alpha}\right)$ & $D T_{0}$ & $A$ & $B$ & $T_{g}(P)$ \\
\hline \multirow{6}{*}{ PPGE } & 214.5 & 293 & 4.8 & \# & 18.6 & 854 & 293 \\
\hline & 132.8 & 283 & 3.6 & $\#$ & 23 & 793 & 283 \\
\hline & 34.3 & 268 & 0.2 & $\#$ & 4.8 & 165 & 223 \\
\hline & 0.1 & 263.8 & 14.4 & 3.3 & \# & 4.6 & 263.8 \\
\hline & 298 & 303 & 12.1 & 2.6 & \# & 3.9 & 303 \\
\hline & 500 & 327 & 9.1 & 1.2 & \# & 3.4 & 327 \\
\hline \multirow{4}{*}{ DGEBA } & 244 & 293 & 6.1 & 15.2 & $\#$ & 763.1 & 293 \\
\hline & 174.3 & 283 & 5.0 & 26.5 & \# & 935 & 283 \\
\hline & 0.1 & 258 & 13.9 & \# & 2.6 & 3.76 & 258 \\
\hline & 400 & 312 & 15.9 & \# & 3.57 & 4.69 & 312 \\
\hline
\end{tabular}

the glass transition occurs at higher temperature, consequently the supercooled liquid state is characterized by higher temperature than at ambient pressure. As an effect of the increase of pressure and temperature, supercooled liquids have larger density and molecules are affected by larger thermal agitation. Such combined effects led to an overall reduction of $\Delta \varepsilon_{\alpha}$ evidencing that the effect of thermal agitation prevails on the increase of density. Since the secondary relaxation is here investigated in the glassy state, where molecular motion are greatly hindered, the effect of thermal agitation on such process are minor, and $\Delta \varepsilon_{\beta}$ remain almost constant or slightly increase. Then the decrease of the ratio $\Delta \varepsilon_{\alpha} / \Delta \varepsilon_{\beta}$ could be interpreted as a consequence of thermal agitation mainly acting on the structural process.

\section{CONCLUSION}

The structural $\alpha$-relaxation of glass-forming substances is naturally a many-body process because of intermolecular interactions and constraints. Therefore properties of the $\alpha$-relaxation originate from many-body dynamics or are directly related to the intermolecular potential and are unsurprisingly correlated with each other. Results from molecular dynamics simulations and experiments show close relations between the Johari-Goldstein (JG) $\beta$-relaxation and the structural $\alpha$-relaxation in their relaxation times and in various properties, indicating that they are not independent of each other. We investigated the relation between secondary and structural dynamics of polyphenylglycidy-ether (PPGE), diglycidyl-ether of bisphenol-A (DGEBA) under variations of temperature and pressure. The investigation of the ratio of dielectric strength of two processes close to $T_{g}$ evidence that the importance of secondary dynamics in relaxing external electric stresses increase in glass formers at high pressures with respect to that of the structural relaxation. We suggest that the thermal agitation, acting above $T_{g}$ is at the basis of the observed result.

\section{REFERENCES}

[1] Kessairi, K., Capaccioli, S., Prevosto, D., Lucchesi, M., Sharifi, S. and Rolla, P.A. (2008). Interdependence of primary and Johari-Goldstein secondary relaxations in glass-forming systems. Journal of Chemical Physics, 112, 4470-4473. doi:10.1021/jp800764w

[2] Prevosto, D., Sharifi, S., Capaccioli, S., Rolla, P., HenselBielowka, S. and Paluch, M. (2007) New experimental evidences about secondary processes in phenylphthaleindimethylether and 1,1'-bis(p-methoxyphenyl)-cyclohexane. Journal of Chemical Physics, 127, 114507. doi:10.1063/1.2771169

[3] Struik, L.C.E. (1978) Physical aging in amorphous polymers and other materials. Elsevier, Amsterdam.

[4] Prevosto, D., Capaccioli, S., Lucchesi, M., Rolla, P.A. and Ngai, K.L. (2004) Dynamics of supercooled and glassy dipropyleneglycol dibenzoate as functions of temperature and aging: Interpretation within the coupling model framework. Journal of Chemical Physics, 120, 4808. doi:10.1063/1.1646375

[5] Johari, G.P. (1982) Effect of annealing on the secondary relaxations in glasses. Journal of Chemical Physics, 77, 4619. doi:10.1063/1.444414

[6] Etienne, S., David, L., Duval, E., Mermet, A., Wypych, A. and Simeoni, G. (2006) Physical aging of amorphous matter: Down to the nanometric scale. Solid State Phenomena, 115, 99. doi:10.4028/www.scientific.net/SSP.115.99

[7] Wypych, A., Duval, E., Boiteux, G., Ulanski, J., David, L. 
and Mermet, A. (2005) Effect of physical aging on nanoand macroscopic properties of poly(methyl methacrylate) glass. Polymer, 46, 12523. doi:10.1016/j.polymer.2005.10.116

[8] Bree, H.W., Heijboer, J., Struik, L.C.E. and Tak, A.G.M. (1974) The effect of densification on the mechanical properties of amorphous glassy polymers. Journal of Polymer Science: Polymer Physics Edition, 12, 1857. doi:10.1002/pol.1974.180120909

[9] Kovacs, A.J., Stratton, R.A. and Ferry, J.D. (1963) Dynamic mechanical properties of poly(vinyl acetate) in shear in the glass transition temperature range. Journal of Chemical Physics, 67, 152-161.

[10] McKinney, J.E. and Goldstein, M. (1974) PVT relationships for liquid and glassy poly(vinyl acetate). Journal of Research of the National Bureau of Standards, Section A, 78A, 331.

[11] Olsen, N.B., Christensen, T. and Dyre, J.C. (2000) $\beta$ relaxation of nonpolymeric liquids close to the glass transition. Physical Review E, 62, 4435. doi:10.1103/PhysRevE.62.4435

[12] Reiser, A., Kasper, G. and Hunklinger, S. (2004) Effect of pressure on the secondary relaxation in a simple glass former. Physical Review Letters, 92, 125701.

[13] Paluch, M., Pawlus, S., Hensel-Bielowka, S., Kaminski, K., Psurek, T., Rzoska, S.J., Ziolo, J. and Roland, C.M. (2005) Effect of glass structure on the dynamics of the secondary relaxation in diisobutyl and diisoctyl phthalates. Physical Review B, 72, 224205. doi:10.1103/PhysRevB.72.224205

[14] Prevosto, D., Capaccioli, S., Lucchesi, M., Rolla, P.A., Paluch, M. and Pawlus, S. (2006) Effect of thermodynamic history on secondary relaxation in glassy phenolphthalein-dimethyl-ether. Physical Review B, 73, 104205. doi:10.1103/PhysRevB.73.104205

[15] McCrum, N.G., Read, B.E. and Williams, G. (1967) Anelastic and dielectric effects in polymeric solids. Wiley, New York.

[16] Johari, G.P. and Goldstein, M. (1970) Viscous liquids and the glass transition. II. Secondary relaxations in glasses of rigid molecules. Journal of Chemical Physics, 53, 2372. doi:10.1063/1.1674335

[17] Ngai, K.L. (2003) An extended coupling model description of the evolution of dynamics with time in supercooled liquids and ionic conductors. Journal of Physics: Condensed Matter, 15, S1107. doi:10.1088/0953-8984/15/11/332

[18] Ngai, K.L. and Paluch, M. (2004) Classification of secondary relaxation in glass-formers based on dynamic properties. Journal of Chemical Physics, 120, 857. doi:10.1063/1.1630295

[19] Sharifi, S. (2011) Temperature dependence of the activation volume of secondary relaxation in glass formers.
ISRN Materials Science, 460751.

[20] Sharifi, S. and Asl, J.M. (2011) Secondary relaxation inside the glass. ISRN Materials Science, 201, 764874.

[21] Sharifi, S. (2011) Activation volume of secondary relaxation. Materials Sciences and Applications, 2, 624-628. doi:10.4236/msa.2011.26084

[22] Ngai, K.L. (2003) An extended coupling model description of the evolution of dynamics with time in supercooled liquids and ionic conductors. Journal of Physics: Condensed Matter, 15, S1107. doi:10.1088/0953-8984/15/11/332

[23] Prevosto, D., Capaccioli, S., Sharifi, S., Kessairi, K. Lucchesi, M. and Rolla, P.A. (2007) Secondary dynamics in glass formers: Relation with the structural dynamics and the glass transition. Journal of Non-Crystalline Solids, 353, 4278-4282. doi:10.1016/j.jnoncrysol.2007.03.045

[24] Paluch, M., Pawlus, S., Hensel-Bielowka, S., Kaminska, E., Prevosto, D., Capaccioli, S., Rolla, P.A. and Ngai, K.L. (2005) Two secondary modes in decahydroisoquinoline: Which one is the true Johari Goldstein process? Journal of Chemical Physics, 122, 234506. doi:10.1063/1.1931669

[25] Sharifi, S., Prevosto, D., Capaccioli, S., Lucchesi, M. and Rolla, P. (2011) Temperature and pressure dependence of secondary process in an epoxy system. Journal of Chemical Physics, 134, 044510. doi:10.1063/1.3518972

[26] Paluch, M., Casalini, R. and Roland, C.M. (2002) Relative contributions of thermal energy and free volume to the temperature dependence of structural relaxation in fragile glass-forming liquids. Physical Review B, 66, 092202. doi:10.1103/PhysRevB.66.092202

[27] Ngai, K.L. and Capaccioli, S. (2004) Relation between the activation energy of the Johari-Goldstein $\beta$ relaxation and Tg of glass formers. Physical Review E, 69, 031501. doi:10.1103/PhysRevE.69.031501

[28] Paluch, M., Patkowski, A. and Fisher, E.W. (2000) Temperature and pressure scaling of the $\alpha$ relaxation process in fragile glass formers: A dynamic light scattering study. Physical Review Letters, 85, 2140. doi:10.1103/PhysRevLett.85.2140

[29] Paluch, M., Gapinski, J., Patkowski, A. and Fischer, E.W. (2001) Does fragility depend on pressure? A dynamic light scattering study of a fragile glass-former. Journal of Chemical Physics, 114, 8048. doi:10.1063/1.1362293

[30] Comez, L., Fioretto, D., Palmieri, L., Verdini, L., Rolla, P.A., Gapinski, T., Pakula, A., Patkowski, W.S. and Fischer, E.W. (1999) Light-scattering study of a supercooled epoxy resin. Physical Review E, 60, 3086. doi:10.1103/PhysRevE.60.3086

[31] Paluch, M., Roland, C.M., Gapinski, J. and Patkowski, A. (2003) Pressure and temperature dependence of structural relaxation in diglycidylether of bisphenol A. The Journal of Chemical Physics, 118, 3177. doi:10.1063/1.1538597 